\title{
Tracking Objects with Shadows
}

\author{
Hao Jiang and Mark S. Drew \\ School of Computing Science, \\ Simon Fraser University, Vancouver, Canada
}

\begin{abstract}
In this paper, we present a new method for tracking objects with shadows. Traditional motion-based tracking schemes cannot usually distinguish the shadow from the object itself, and this results in a falsely captured object shape. If we want to utilize the object's shape information for a pattern recognition task, this poses a severe difficulty. In this paper we present a color processing scheme to project the image into an illumination invariant space such that the shadow's effect is greatly attenuated. The optical flow in this projected image together with the original image is used as a reference for object tracking so that we can extract the real object shape in the tracking process. We present a modified snake model for general video object tracking. A new external force is introduced into the snake equation based on the predictive contour such that the active contour is attracted to a shape similar to the one in the previous video frame. The proposed method can deal with the problem of an object's ceasing movement temporarily, and can also avoid the problem of the snake tracking into the object interior. Global affine motion estimation is applied to eliminate the effect of camera motion and hence the method can be applied in a general video environment. Experimental results show that the proposed method can track the real object even if there is strong shadow influence.
\end{abstract}

\section{INTRODUCTION}

Shadows usually present a confounding factor for correct object tracking. Traditional motion detection schemes cannot distinguish the moving object and the shadows moving with it. Therefore object tracking results based on traditional schemes usually result in a combination of the object and its shadow. This kind of result will pose severe difficulty if the contour is further passed to an analyzer for object recognition. Eliminating the shadow and tracking the real contour of an object is a challenging problem. Different schemes have been presented to try to attenuate the shadow's influence in applications such as object tracking and still image segmentation. In Ref. 1 the shadow detection and elimination problem is studied in the context of road human surveillance. In the specific application, the lighting condition is restricted to sunlight; the internal and external parameters of the video camera are fixed and the target objects are restricted to a walking human being. The paper presented a method of locating the real position of a walking human by extracting the core lines of human and the core lines of the shadows based on the motion detection map. In Ref. 2 a geometrical scheme based on stereo vision is presented for shadow elimination in surveillance video tracking. The scheme is based on image subtraction. The image captured by the first camera is first projected onto the road plane and then further projected to the image plane of the second camera. The road maps of two images will map perfectly while the other parts such as walking humans will not map well. Using the second image to subtract the projection of the first image will eliminate everything on the road plane including the moving shadows. In Ref. 3, simple illumination invariant features are applied so as to get an image which reflects the difference of surface materials. Since the cast shadow only changes the illumination of backgrounds, the illumination invariant features will attenuate the shadow effects. The method is applied in the problem of still image segmentation. In Ref. 4, a statistics method is presented for pixel classification. The features used include the illuminance and normalized chrominance vector. The color changing of a pixel is described by multiplying some constant in each color channel. Pixels are classified into three classes: background, foreground and shadow, based on maximum posterior classification. Spatial information is also applied to improve the dense region classification result. In Ref.5, the shadow detection problem is studied based on a model similar to the Phong model. Heuristic methods are presented to classify the shadow and foreground object.

Further author information: (Send correspondence to M.S.D.)

E-mail: \{hjiangb,mark\}@cs.sfu.ca, Telephone: 1604291 4682,

Web: www.cs.sfu.ca/ $\sim$ mark 
In this paper, we present a different method based on an illumination invariant color space and an inertia enhanced snake model for reliable object tracking in a general video environment. If lighting is approximately Planckian, then in Wien's approximation the resulting simple exponential form of the illumination spectrum leads to the conclusion that as temperature $T$ changes, characterizing the illumination color, a $\log -\log$ plot of 2-dimensional $\{\log (\mathrm{R} / \mathrm{G}), \log (\mathrm{B} / \mathrm{G})\}$ values for any single surface forms a straight line provided camera sensors are fairly narrow-band. ${ }^{6-8}$ Thus lighting change reduces to a linear transformation along an almost straight line, even for real data with only approximately Planckian lighting. For many a target with many paint patches, mean-subtracted log-log plots all cluster around a single line through the origin that characterizes lighting change. The invariant image is thus the gray scale image that results from projecting $\log -\log$ pixel values onto the direction orthogonal to lighting change, within and outside the umbra; the projection greatly attenuates shadowing. Based on this color projection, we further present an inertia enhanced snake model for tracking objects with shadows. In our model, an additional energy term is included, based on the predictive contour. This term attracts the active contour to converge to a shape similar to the one in the previous video frame. As well, instead of simply using the predictive contour to re-initialize the snake, we construct a new initial contour by a uniform expansion along the normal of the previous contour. This scheme prevents the contour from erroneously tracking features inside the true boundary of the object. At the same time, the new predictive contour inertia energy term makes the snake ignore distracting elements. As well, if the object stops moving temporarily, the snake will evolve according to the inertia term in the predictive contour and converge to a shape that corresponds to the motion prediction result. We adopt an affine motion model for global motion estimation and camera motion compensation with the result that our scheme can work in a general video environment.

The organization of the paper is as follows. We first study shadow invariant image space in section 2 . We show that under Planckian lighting, the $\log -\log$ ratio of $(\log (R / G), \log (B / G))$ forms a straight line for different materials for narrow-band sensor cameras. We further present a camera calibration scheme for shadow invariant image generation in section 2.1. In section 3, we present the tracking schemes based on an inertia snake model and shadow invariant image for shadow resistant video tracking. The modified snake equation is studied in section 3.1. The contour prediction and smoothing schemes are presented in 3.2. In section 3.3, we show how to generate external forces based on global motion compensated motion detection and gradient vector flow. Experiments, results, and discussions are presented in section 4.

\section{SHADOW INVARIANT IMAGE SPACE}

Considering a flat surface illuminated by $i=1 \ldots L$ lights. The spectrum of the $i$ th Planckian lighting source can be well approximated as

$$
E_{i}(\lambda)=I_{i} c_{1} \lambda^{-5} e^{-\frac{c_{2}}{\lambda T}}
$$

with direction $\mathbf{a}_{i}$, where $I_{i}$ is the intensity of the lighting source, $T$ is temperature, $c_{1}$ and $c_{2}$ are constants. We assume that the sensors of the image capturing device are narrow-band such that they can be well approximated with spike sensitivities $Q_{k}(\lambda)=q_{k} \delta\left(\lambda-\lambda_{k}\right), k=1,2,3$, in which case we only consider common three-sensor cameras. But our result can be easily extended to the situation where there are more sensors. We further ignore the color response change of a point with respect to the viewing angle. Based on the above assumption, the sensor response at point $\mathbf{x}$ corresponding to sensor $k$ under illumination $i=1 \ldots L$ is,

$$
\begin{aligned}
\rho_{k}(\mathbf{x}) & =\sum_{i=1}^{L}\left[\int_{0}^{+\infty} E_{i}(\lambda) S(\lambda) Q_{k}(\lambda) d \lambda\right] \\
& =\left[\sum_{i=1}^{L} c_{1} \mathbf{a}_{i} \cdot \mathbf{n}(\mathbf{x}) I_{i}\right] S\left(\lambda_{k}\right) \lambda_{k}^{-5} e^{-\frac{c_{2}}{\lambda_{k} T}} q_{k}
\end{aligned}
$$

where $\mathbf{n}(\mathbf{x})$ is the normal vector of the object surface at point $\mathbf{x}$, corresponding to surface spectral reflectance function $S(\lambda)$. For three-sensor cameras, we calculate the $\log$-ratios $r=\log \left[\rho_{1} / \rho_{2}\right]$ and $w=\log \left[\rho_{3} / \rho_{2}\right]$ and get a linear relationship between this two ratios for a given reflectance surface, 


$$
w-\log \left(\frac{q_{3} S\left(\lambda_{3}\right) \lambda_{2}^{5}}{q_{2} S\left(\lambda_{2}\right) \lambda_{3}^{5}}\right)=\left[r-\log \left(\frac{q_{1} S\left(\lambda_{1}\right) \lambda_{2}^{5}}{q_{2} S\left(\lambda_{2}\right) \lambda_{1}^{5}}\right)\right] \frac{\lambda_{3} \lambda_{1}-\lambda_{2} \lambda_{1}}{\lambda_{1} \lambda_{3}-\lambda_{2} \lambda_{3}}
$$

From Eq.3, each reflectance surface is characterized by a fixed line. Every such line, for a particular camera, has the same slope, determined by the sensors in the log-log-ratio space under different illuminations. The orientation is defined as the characteristic orientation of the camera. To distinguish between different materials, we can just project the lines to the orientation orthogonal to the camera characteristic orientation to get an illumination invariant image. In the following paragraphs we only consider three-sensor cameras; we assume $\rho_{1}=R, \rho_{2}=G, \rho_{3}=B$ and $r=\log [R / G]$, $w=\log [B / G]$.

\subsection{Camera Calibration}

Because different cameras have different color characteristics, they must be calibrated before using in our tracking scheme. In this section, we study calibrating the camera for illumination-invariant image generation. For real cameras, the characteristic curves of sensors are not strictly delta functions, and Eq. 3 is thus an approximation for narrow-band cameras. In practical situations, the log-log ratio plot corresponding to one material under different lighting is not strictly a line. For different materials the log-log ratio plots generally have some fixed dominant orientation. From Eq. 3, the dominant orientation is only a function of camera sensor center frequencies and does not correlate with the lighting condition and the surface material. Therefore the calibration process for a specific camera just needs to be done once and can be used for different conditions.

Assume $P$ is the collection of $\log$-log ratio pair set $\left\{\left(r_{i}^{k}, w_{i}^{k}\right) \mid i \in I, k \in K\right\}$ where $r_{i}^{k}=\log \left(R_{i}^{k} / G_{i}^{k}\right)$ and $w_{i}^{k}=$ $\log \left(B_{i}^{k} / G_{i}^{k}\right) ;\left(R_{i}^{k}, G_{i}^{k}, B_{i}^{k}\right)$ is the color of pixel $i$ under illumination $k$ in the RGB color space; $I$ and $K$ are the whole set of pixel indexes and illumination indexes respectively. We first shift the log-log ratio vector such that the center of the cluster corresponding to one pixel under different illuminations is located at the origin of the coordinate system. The center alignment $\log -\log$ ratio set is denoted as $\left\{\left(\hat{r}_{i}^{k}, \hat{w}_{i}^{k}\right) \mid i \in I, k \in K\right\}$, where

$$
\begin{aligned}
\hat{r}_{i}^{k} & =r_{i}^{k}-\frac{1}{|K|} \sum_{k \in K} r_{i}^{k} \\
\hat{w}_{i}^{k} & =w_{i}^{k}-\frac{1}{|K|} \sum_{k \in K} w_{i}^{k}
\end{aligned}
$$

Now we can just strip out the indices and denote the center-shifted log-log ratio pair set by $\{(r, w)\}$. The crosscorrelation matrix $C$ of the center shifted $\log -\log$ ratio pair set is calculated by

$$
C=\left[\begin{array}{cc}
E_{r r} & E_{r w} \\
E_{w r} & E_{w w}
\end{array}\right]
$$

where $E_{r r}=E\left[r^{2}\right], E_{w w}=E\left[w^{2}\right], E_{r w}=E_{w r}=E[r w]$. Let $\mu_{1}$ and $\mu_{2}$ be eigenvalues of $C$, where

$$
\begin{aligned}
& \mu_{1}=\frac{1}{2} E_{r r}+\frac{1}{2} E_{w w}+\frac{1}{2} \sqrt{\left(E_{r r}^{2}-2 E_{r r} E_{w w}+E_{w w}^{2}+4 E_{r w}^{2}\right)} \\
& \mu_{2}=\frac{1}{2} E_{r r}+\frac{1}{2} E_{w w}-\frac{1}{2} \sqrt{\left(E_{r r}^{2}-2 E_{r r} E_{w w}+E_{w w}^{2}+4 E_{r w}^{2}\right)}
\end{aligned}
$$

Then the eigenvector $v_{1}$ corresponding to $\mu_{1}$ is defined as the principle orientation of the camera.

$$
v_{1}=\left(-\frac{-\frac{1}{2} E_{r r}+\frac{1}{2} E_{w w}-\frac{1}{2} \sqrt{\left(E_{r r}^{2}-2 E_{r r} E_{w w}+E_{w w}^{2}+4 E_{r w}^{2}\right)}}{E_{r w}}, 1\right)^{T}
$$

Its orthogonal vector is

$$
v_{2}=\left(-\frac{-\frac{1}{2} E_{r r}+\frac{1}{2} E_{w w}+\frac{1}{2} \sqrt{\left(E_{r r}^{2}-2 E_{r r} E_{w w}+E_{w w}^{2}+4 E_{r w}^{2}\right)}}{E_{r w}}, 1\right)^{T}
$$

The invariant image is calculated as the grayscale image

$$
I M=\frac{(\log [R / G], \log [B / G])}{\left\|v_{2}\right\|} v_{2}
$$




\section{AN INERTIA SNAKE MODEL}

\subsection{Snake Equation with Predictive Contour Inertia}

In this section, we present the equation for enhancing the robustness of active contours ${ }^{9}$ in the tracking problem. We formulate a new snake equation ${ }^{10}$ :

$$
\min _{X(s)} \int_{s} \frac{\alpha}{2}|\nabla X(s)|^{2}+\frac{\beta}{2}\left|\nabla^{2} X(s)\right|^{2}+P(X(s))+\frac{\gamma}{2} E(X(s), C(s)) d s
$$

where $X(s): R \rightarrow R^{2}$ is the active contour of current frame, $C(s): R \rightarrow R^{2}$ is the prediction contour from the previous frame. $E(X(s), C(s))$ is a term which measures the difference between the $X(s)$ and $C(s)$. Just as traditional snake, the internal energy of the active contour is introduced by the term $\frac{\alpha}{2}|\nabla X(s)|^{2}+\frac{\beta}{2}\left|\nabla^{2} X(s)\right|^{2} . P(X(s))$ is the external force based on the feature of interest such as the edges or the motion of the object.

Before discussing the formulation of $E(X(s), C(s))$, it should be noted that $s$ is not necessarily the length of the contour in the previous frame. On the other hand, it should be viewed as the normalized length in the range of $[0,1)$ by the contour length in each frame. We will see that this does not pose a problem in the numerical implementation if we fix the number of the nodes on the contour. In this case, the parameter of $s$ is implicitly normalized.

One natural choice for $E(X(s), C(s))$ is

$$
E(X(s), C(s))=\|X(s)-C(s)\|
$$

If the norm is chosen as the quadratic norm, the corresponding Euler Equation is

$$
\alpha X_{s s}-\beta X_{s s s}-\nabla P(X)+\gamma(C-X)=0
$$

and the steepest descent solution is

$$
\frac{\partial X}{\partial t}=\alpha X_{s s}-\beta X_{s s s s}-\nabla P(X)+\gamma(C-X)
$$

We generalize the above equation and substitute $-\nabla P(X)$ by $F_{e x t}(X)$,

$$
\frac{\partial X}{\partial t}=\alpha X_{s s}-\beta X_{s s s s}+F_{e x t}(X)+\gamma(C-X)
$$

Now we get the modified active contour for the problem of tracking. We introduce another force term based on the prediction contour. After selecting weight $\gamma$, we can control the degree the prediction has on the contour tracking.

\subsection{Contour Prediction and Smoothing}

We predict the future contour position and shape by the method of block-wise motion estimation. For every point $(x, y)$ on the previous contour $Y$, a square block width of $d$ is constructed centered on the pixel. By setting a searching window $w$, the best matching block center is selected as the predicted point.

$$
\begin{aligned}
& (\widehat{\Delta x}, \widehat{\Delta y})=\min _{\Delta x \in(-w / 2, w / 2), \Delta y \in(-w / 2, w / 2)} \int_{\xi=x-d / 2}^{x+d / 2} \int_{\eta=y-d / 2}^{y+d / 2}|u(\xi+\Delta x, \eta+\Delta y, t)-u(\xi, \eta, t-\Delta t)| d \xi d \eta \\
& C=Y+(\widehat{\Delta x}, \widehat{\Delta y})
\end{aligned}
$$

where $u$ is the image sequence. Motion estimation sometimes fails to estimate the correct future contour position. This will occur if some part of the previous contour does not fall at the boundary of an object. This situation is very common for snake tracking of objects with concave boundaries. Another situation is when the object is passing through background edges. Motion estimation sometimes fails to follow the true object boundary but stays with the background edges. To reduce the error of motion estimation, some global motion model can be used. This approach can be viewed as a smoothing process to the motion estimation result. We propose a different approach for smoothing the prediction contour - the smoothing process is a self-evolving curve without the external force: 


$$
\frac{\partial C}{\partial t}=\alpha_{0} C_{s s}-\beta_{0} C_{s s s s}
$$

A stopping time has to be specified so that the curve will not distort too much while smoothing the singular points. One problem of the smoothing process is that the contour shrinks during the process of smoothing. Therefore, we cannot use the prediction contour as the initial contour for the next frame tracking. The initial contour $B$ is thus selected as a uniform expansion of the previous contour tracking result:

$$
B=Y-c \mathbf{n}
$$

where $\mathbf{n}$ is the inwards normal of $Y$ and $c$ is a constant. It is clear that this scheme can work for any motion of the contour with speed less than $c \cdot f$ in the normal direction, where $f$ is the video frame rate.

\subsection{Global Motion Compensation and External Force Formation}

To remove the motion introduced by the camera which is called global motion, we need to estimate the motion in the whole picture. In most circumstances, an affine model is a reasonable approximation. The affine flow field can be represented as

$$
\begin{aligned}
& \theta_{1}(x, y)=p_{1}+p_{2} x+p_{3} y \\
& \theta_{2}(x, y)=p_{4}+p_{5} x+p_{6} y
\end{aligned}
$$

where $\mathbf{p}=\left\{p_{1}, p_{2}, p_{3}, p_{4}, p_{5}, p_{6}\right\}^{T}$ are the parameters to be estimated. This can be written in matrix notation as

$$
\theta(x, y)=A(x, y) \mathbf{p}
$$

where

$$
A(x, y)=\left(\begin{array}{cccccc}
1 & x & y & 0 & 0 & 0 \\
0 & 0 & 0 & 1 & x & y
\end{array}\right)
$$

The optical flow equation can be written as

$$
\nabla u^{T}(A \mathbf{p})+u_{t}=0
$$

This is an over-determined problem, in that we have more equations than the number of the unknowns. We can use Least Square Error model to get an approximation solution, which is formulated as minimizing $E$ defined by

$$
E=\sum_{x, y}\left(\nabla u^{T}(A \mathbf{p})+u_{t}\right)^{2}
$$

By taking the derivative of $E$ with respect to $\mathbf{p}$ and setting it to zero, we get the solution for affine parameters,

$$
\mathbf{p}=\left(\sum A^{T} \nabla u \nabla u^{T} A\right)^{-1} \sum\left(-A^{T} \nabla u u_{t}\right)
$$

In our tracking scheme, two motion detection maps are generated. The first one is based on the original video with global motion compensated. The other one is based on the shadow invariant global motion compensated images. We then use a simple thresholding scheme to detect the motion feature in both sequences (more complex models including optical flow could be incorporated into the algorithm). The intersection of both these motion detecting results produces an image segmentation map which is used to calculate the external force field of the snake model based on the Gradient Vector Flow scheme. ${ }^{11}$ 


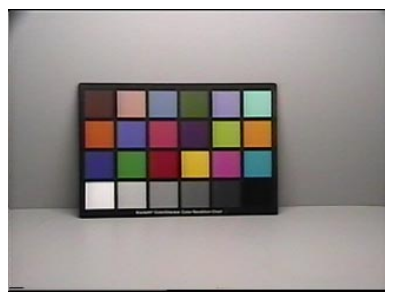

(a) Illumination 1 .

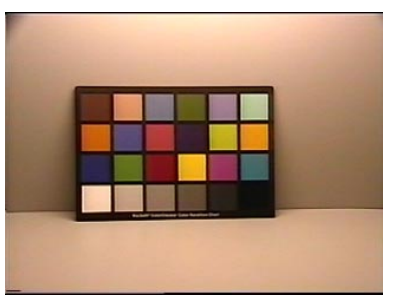

(b) Illumination 2 .

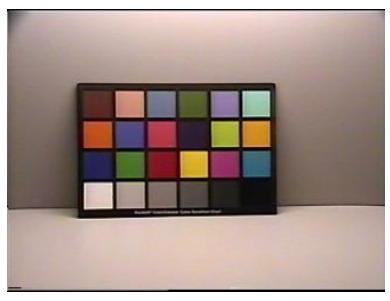

(c) Illumination 3 .

Figure 1. The color chipboards used for camera calibration. [Color images may be viewed at www.cs.sfu.ca/ mark/ftp/Spie03/spie03.pdf]

\subsection{Numerical Implementation}

We follow the finite difference method to discretize Eq. 11. Let

$$
X_{i}^{n}=\left(x_{i}^{n}, y_{i}^{n}\right)=(x(i \Delta s, n \Delta t), y(i \Delta s, n \Delta t))
$$

The numerical form for Eq. 11 is

$$
\begin{aligned}
\frac{X_{i}^{n+1}-X_{i}^{n}}{\Delta t}= & \frac{\alpha}{\Delta s^{2}}\left[X_{i+1}^{n+1}+X_{i-1}^{n+1}-2 X_{i}^{n+1}\right]- \\
& \frac{\beta}{\Delta s^{4}}\left\{\left[X_{i+2}^{n+1}+X_{i}^{n+1}-2 X_{i+1}^{n+1}\right]-2\left[X_{i+1}^{n+1}+X_{i-1}^{n+1}-2 X_{i}^{n+1}\right]+\left[X_{i}^{n+1}+X_{i-2}^{n+1}-2 X_{i-1}^{n+1}\right]\right\} \\
& +F_{\text {ext }}\left(X_{i}^{n}\right)+\gamma\left(C_{i}^{n}-X_{i}^{n}\right)
\end{aligned}
$$

Let $x^{n}=\left[x_{0}^{n}, x_{1}^{n}, \ldots, x_{N-1}^{n}\right]^{T}, y^{n}=\left[y_{0}^{n}, y_{1}^{n}, \ldots, y_{N-1}^{n}\right]^{T}$ and $X^{n}=\left[x^{n}, y^{n}\right]$, where $N$ is the number of nodes in the discrete contour. Construct band matrix $A$ as,

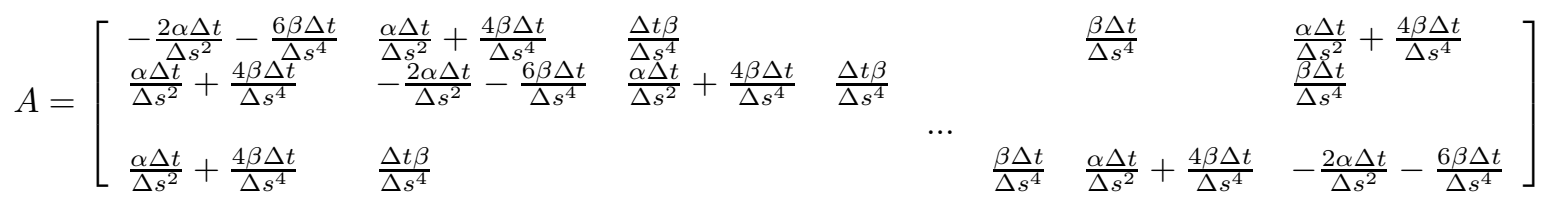

We have,

$$
X^{n+1}-X^{n}=A X^{n+1}+\Delta t F_{e x t}\left(X^{n}\right)+\Delta t \gamma\left(C^{n}-X^{n}\right)
$$

Solving for $X^{n+1}$,

$$
X^{n+1}=(I-A)^{-1}\left[X^{n}+\Delta t F_{\text {ext }}\left(X^{n}\right)+\Delta t \gamma\left(C^{n}-X^{n}\right)\right]
$$

Eq. 12 is a simplified version and can also be discretized by the above methods. 


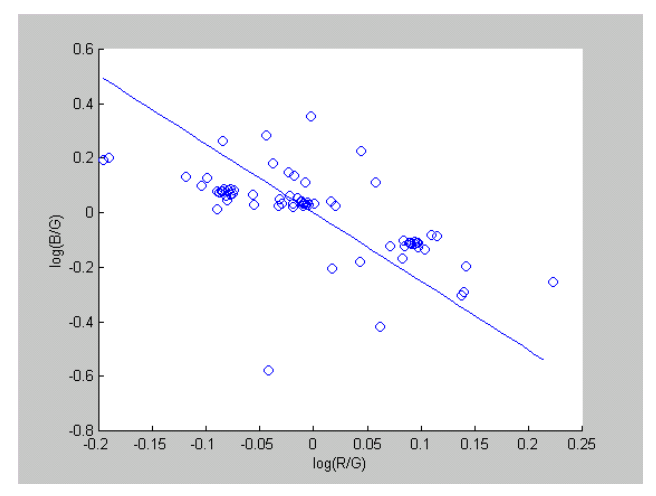

Figure 2. Camera calibration to find characteristic orientation.

\section{EXPERIMENT RESULTS}

We use a consumer camcorder (Canon ES60) in our experiment - we did not use a specially selected camcorder in order to show the robustness of our method for general environments. Before the tracking experiment, we first calibrated the camcorder and got the shadow invariant orientation. We used a color chipboard of 24 colors under three standard illuminations for the camera calibration. The images for the calibration are shown in Fig. 1.

We then captured camcorder images of the color board under three different illuminations which include daylight and two different indoor lightings. During the video capturing process, both the camera and the chipboard were fixed such that we could retain the pixel correspondence under different illuminations. The video was then further digitized into digital images. We manually segmented the images into regions corresponding to different color blocks. The color in each region is represented by the mean $\mathrm{R}, \mathrm{G}$ and $\mathrm{B}$ value. Fig 2 shows that a scatter plot of the center-shifted log-log ratio data gives the illumination invariant orientation of the camcorder. The orientation vector measured was [0.37, -0.93]. The center-shifted $\log -\log$ ratios corresponding to each material are well fit to the straight line. Only a few measurement data have relatively large errors. The error is caused by several factors. One of them is the model error. Since the straight line model is only exact for the ideal pulse sensor cameras, the real wide-band sensors will cause dispersion of the log-log ratios. Other errors come from the measuring limitations posed by the camera's dynamic range and digitizer's quantization effect. As shown in the following experiment, the estimated orientation is robust enough to greatly improve the tracking result.

Fig 3 shows the motion detection result for a two-color ball rolling on the ground based on the original image sequence and the illumination invariant sequence. The traditional motion detection scheme finds large errors on both the object's boundary and the shadow's boundary. Motion detection based on the shadow invariant image obtains much better results. The shadow's influence is nearly totally removed. We also note that the background of the shadow invariant image motion detection result is also much clearer. To increase the robustness we use the intersection of the motion detection map of the original image sequence and that for the shadow invariant image sequence as the final motion detection map. Fig 4 shows the tracking result for the rolling ball sequence. The result shows that the contour of the ball is well tracked without the distraction of the shadow. Fig 5 shows another experimental result for tracking a hand with shadow under indoor lighting.

\section{CONCLUSION}

We present an efficient algorithm for tracking objects with shadows. The algorithm can eliminate the distracting influence from the shadows and track the shape of the real object. This can be very useful for higher level vision processing such as gesture or behavior recognition. Future research for this project includes speeding up the algorithm such that it can work in real time applications.

\section{REFERENCES}

1. K. Onoguchi, Shadow elimination method for moving object detection, Proceedings of the 14th Int. Conf. on Pattern Recognition 1998 , Vol. 1, 583-587, 1998. 


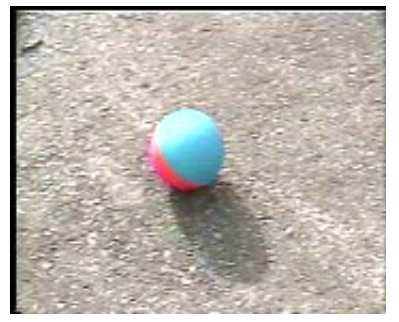

(a)

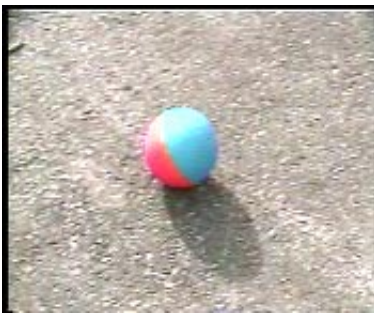

(b)

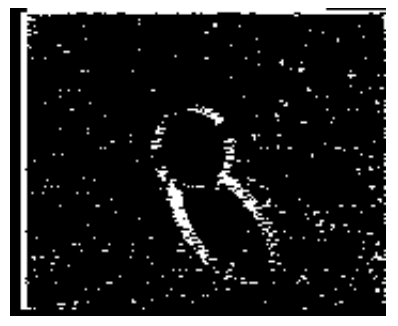

(c)

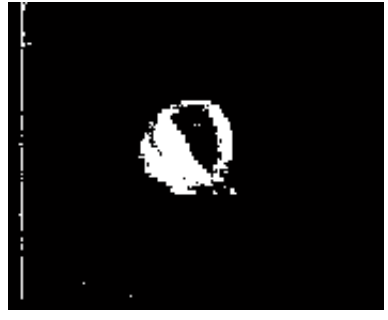

(d)

Figure 3. Motion detection map. (a) Frame one (b) Frame two (c) Motion map by traditional scheme (d) Motion map by shadow invariant scheme.

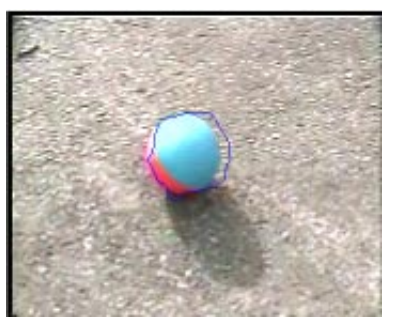

(a) Frame 0

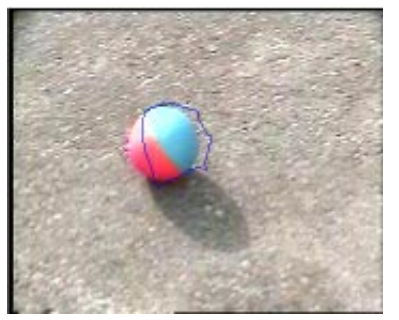

(e) Frame 16

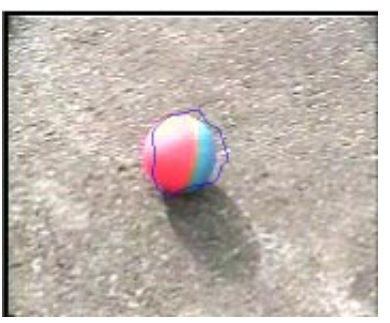

(b) Frame 4

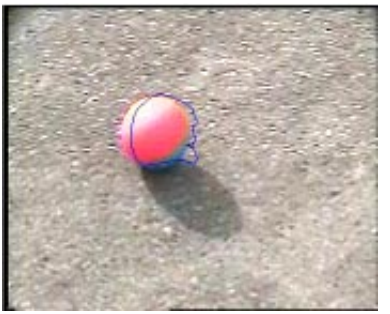

(f) Frame 20

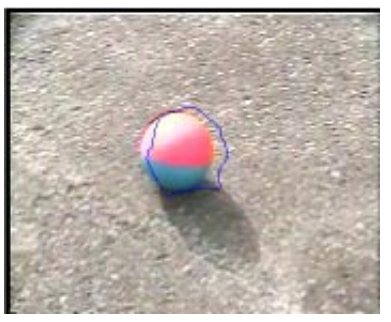

(c) Frame 8

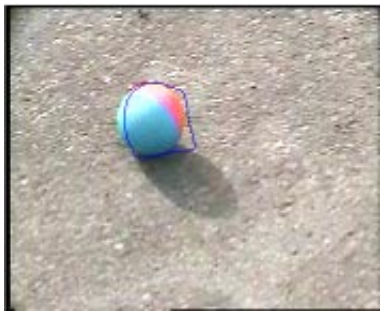

(g) Frame 24

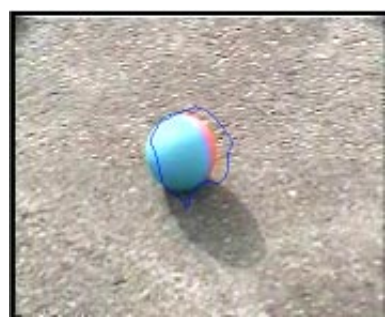

(d) Frame 12

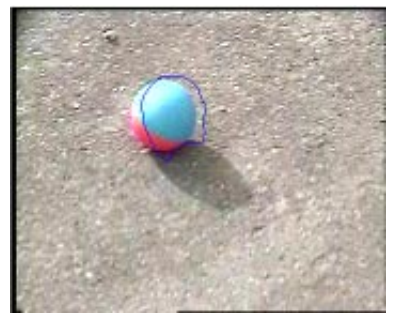

(h) Frame 28

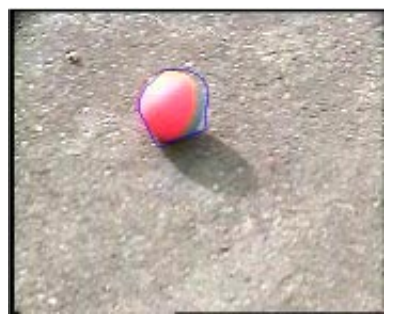

(i) Frame 32

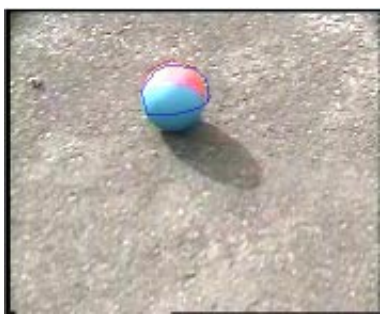

(j) Frame 36

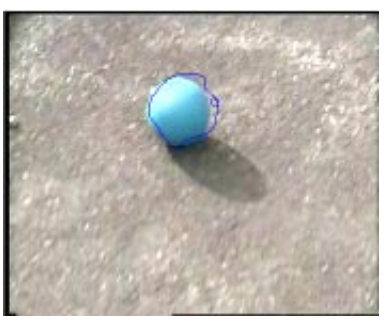

(k) Frame 39

Figure 4. Tracking result with proposed method for the ball sequence. 


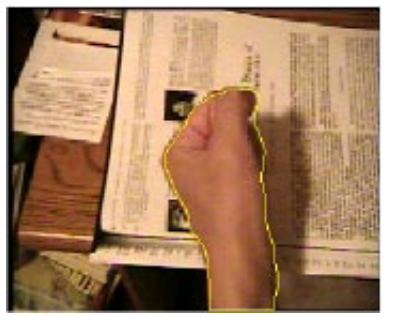

(a) Frame 0

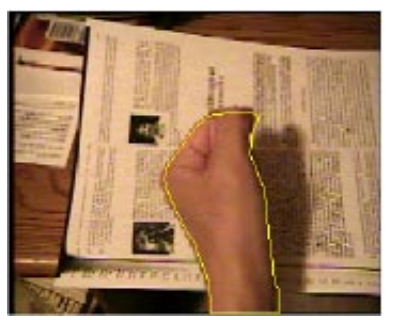

(e) Frame 12

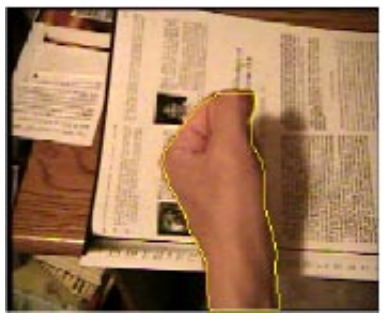

(b) Frame 3

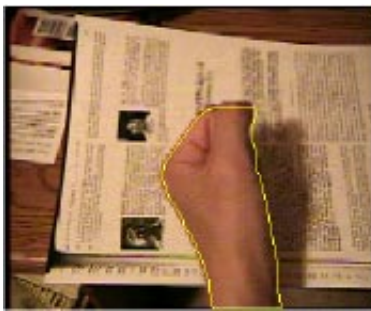

(f) Frame 15

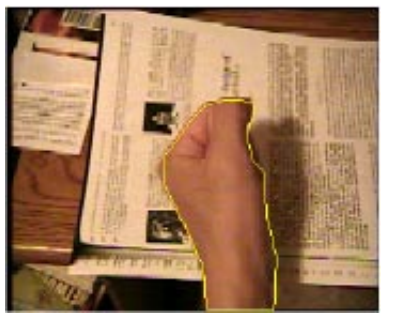

(c) Frame 6

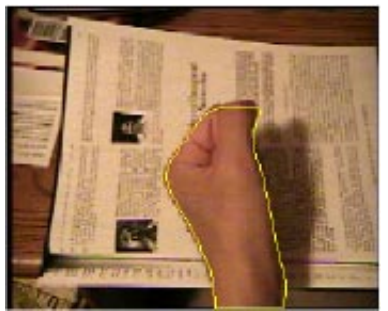

(g) Frame 18

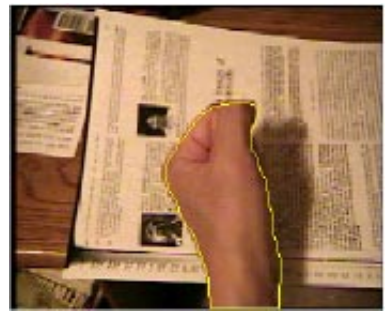

(d) Frame 9

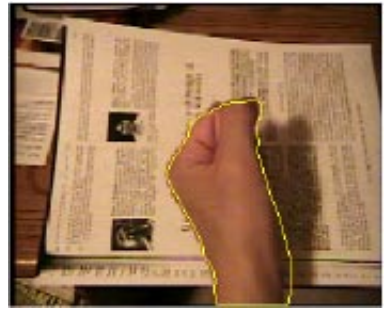

(h) Frame 21

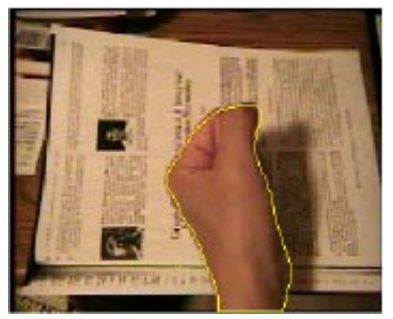

(i) Frame 24

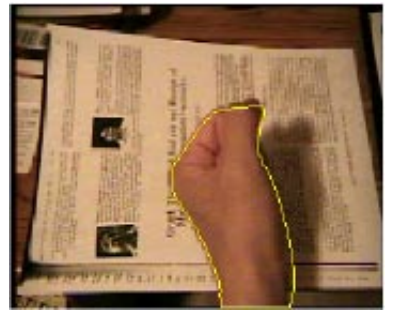

(j) Frame 27

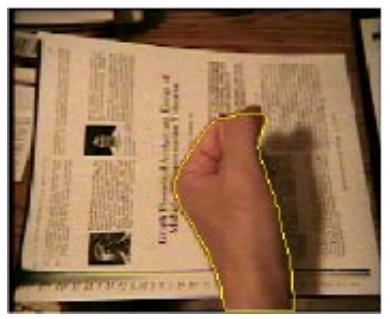

(k) Frame 30

Figure 5. Tracking result with proposed method for the hand sequence. 
2. Y. Sonoda and T. Ogata, Separation of moving objects and their shadows, and application to tracking of loci in the monitoring images, Proceedings of the 4th International Conference on Signal Processing 1998, Vol. 2, 1261-1264, 1998.

3. E. Salvador, A. Cavallaro, and T. Ebrahimi, Shadow identification and classification using invariant color models, ICASSP'01, pp.7-11, 2001.

4. I. Mikic, P. Cosman, G. Kogut, and M. Trivedi, Moving shadow and object detection in traffic scenes, International Conference on Pattern Recognition, September 2000, vol. 1, pp. 321-324.

5. J. Stauder, R. Mech, and J. Ostermann, Detection of moving cast shadows for object segmentation, IEEE Trans. on Multimedia, Vol. 1, No.1, March 1999, pp. 65-76.

6. G.D. Finlayson and S.D. Hordley. Color constancy at a pixel. J. Opt. Soc. Am. A, 18(2):253-264, Feb. 2001. Also, UK Patent application no. 0000682.5. Under review, British Patent Office.

7. G.D. Finlayson and M.S. Drew. 4-sensor camera calibration for image representation invariant to shading, shadows, lighting, and specularities. In ICCV'01: International Conference on Computer Vision, pages II: 473-480. IEEE, 2001.

8. G.D. Finlayson, S.D. Hordley, and M.S. Drew, Removing Shadows from Images, ECCV 2002: European Conference on Computer Vision, 4:823-836, 2002. Lecture Notes in Computer Science Vol. 2353, http://www.cs.sfu.ca/ mark/ftp/Eccv02/shadowless.pdf.

9. M. Kass, A.Wikin, and D. Terzopaulos, Snakes: active contour models, Int. J. Comput. Vision, 1:321-331, 1988.

10. H. Jiang and M.S. Drew, A predictive contour inertia snake model for general video tracking, ICIP'02, 2002, http://www.cs.sfu.ca/ mark/ftp/Icip02a/icip02a.pdf.

11. C. Xu and J.L. Prince, Snake, shapes, and gradient vector flow, IEEE Trans. Image Proc., 7:359-369, 1998. 